\title{
Archives
}

\section{Archives et géographie : typologie, caractéristiques et perspectives}

\section{Laetitia Le Clech}

Volume 47, numéro 1, 2017

URI : https://id.erudit.org/iderudit/1041826ar

DOI : https://doi.org/10.7202/1041826ar

Aller au sommaire du numéro

Éditeur(s)

Association des archivistes du Québec (AAQ)

\section{ISSN}

0044-9423 (imprimé)

2369-9256 (numérique)

Découvrir la revue

\section{Citer cet article}

Le Clech, L. (2017). Archives et géographie : typologie, caractéristiques et perspectives. Archives, 47(1), 59-83. https://doi.org/10.7202/1041826ar

\section{Résumé de l'article}

Dans cet article, Laetitia Le Clech fait le survol de différents enjeux et particularités qui touchent la conservation des archives géographiques. En plus des cartes, ces dernières comprennent également des photographies et images, ainsi que certaines études de terrain. Elle présente dans un premier temps quelques exemples de traitement de documents cartographiques en différents endroits, en plus de nous exposer les défis particuliers que posent ces documents. Les archives géographiques ont de multiples utilités, que ce soit dans le domaine historique, en géopolitique ou même dans le développement de politiques gouvernementales. L'auteure en fait la recension et illustre chacun de ces usages par des exemples. Elle donne ensuite un aperçu de la conservation des documents cartographiques suite à l'avènement de l'ère du numérique. En effet, tout comme pour d'autres types d'archives, ce contexte amène son lot de questionnements et de nouvelles pratiques, que ce soit par la numérisation dans le but de les conserver et de les diffuser, mais aussi par la nécessité de se préoccuper de l'obsolescence des supports et d'établir de nouvelles normes. L'auteure termine son texte en nous présentant diverses initiatives de diffusion et de création qui sont issues de ces nouvelles possibilités qu'offre le monde numérique. Elle ne manque pas en passant de nous mettre en garde contre certains abus et de plaider pour un encadrement renforcé afin de mieux mettre en valeur ces documents bien particuliers.
Ce document est protégé par la loi sur le droit d'auteur. L'utilisation des services d’Érudit (y compris la reproduction) est assujettie à sa politique d'utilisation que vous pouvez consulter en ligne.

https://apropos.erudit.org/fr/usagers/politique-dutilisation/ 


\title{
ÉTUDES
}

\section{Archives et géographie: typologie, caractéristiques et perspectives}

\begin{abstract}
The role of the geographic archive in the formation of a national geography is one of great significance. It keeps the discipline intellectually honest by reference to an original source and not interpretation of secondary source.
\end{abstract}

Geoffrey Martin, historien de la géographie américaine (1964)

\section{LAETITIA LE CLECH}

Diplômée de la maîtrise en sciences de l'information, École de bibliothéconomie et des sciences de l'information (EBSI), Université de Montréal

La discipline géographique commande fréquemment I'utilisation d'archives. En effet, le but de la géographie n'est-il pas d'étudier les milieux naturels et l'humain évoluant dans ces différents milieux? Pour ce faire, on se doit de consulter des documents historiques, iconographiques et scientifiques qui témoignent de l'activité géologique, physique de la Terre et de l'activité culturelle, économique et sociale de l'humain. Les archives géographiques peuvent aussi nous entraîner sur des terrains moins fréquentés, comme la géopolitique, l'environnement et, de plus en plus dans notre société de l'information numérique, servir pour des projets citoyens souvent passionnants. 
Les archives géographiques ont beaucoup évolué dans le temps et nous devons nous ajuster à ces transformations. Qu'en est-il de leur numérisation, de leur pérennité? On peut s'interroger aussi sur le rôle des institutions archivistiques et des archivistes dans la sphère géographique, puisque de plus en plus, les données sont ouvertes à tous, accessibles, et que des applications simples nous permettent parfois de les exploiter.

\section{LA TYPOLOGIE DES ARCHIVES GÉOGRAPHIQUES}

\subsection{Les cartes}

Couture définit la carte comme «une représentation plane d'une partie ou de l'ensemble de la terre ou d'un corps céleste» (1994, p. 218). Les cartes sont des représentations du monde et de l'espace. Utilisée par le géographe, la carte lui permet de situer son objet d'étude et de le représenter. La typologie des archives cartographiques est vaste: ébauches, carnets de notes, photographies aériennes (annotées ou non), cartes manuscrites, fonds de cartes topographiques ou géographiques, cartes copiées à la main, cartes imprimées et cartes reproduites par différents procédés (Rousseau et Couture, 1994, p. 218). On peut y ajouter les plans architecturaux, les croquis, les maquettes, les dessins de présentation, les dessins de perspective, les plans d'aménagement paysager, les documents de compilation et les dessins techniques (Rousseau et Couture, 1994, p. 220). Les documents cartographiques se retrouvent principalement sur des supports papier, mais l'apparition des données géospatiales et le développement de ressources en ligne, accessibles par tous, obligent à une réflexion que nous mènerons plus loin dans ce texte.

Pendant longtemps, les cartes topographiques et géologiques étaient les outils de base des études géographiques, créant une certaine dépendance du géographe vis-à-vis de ses cartes (Ciattoni et Veyret, 2010, p. 263).

\subsection{Les archives iconographiques}

Les documents iconographiques sont souvent utilisés en géographie pour décrire des espaces, caractériser des types de paysages, de 
végétation, de milieux (urbain, rural), etc. Pour les décrire et les manipuler correctement, en particulier les photographies, il est important de bien connaître les différents supports (microfilm, pellicule, photo, etc.) et les différents formats (JPEG, TIFF, PNG, etc.) (Stage technique international des archives, 2010).

La photographie aérienne s'est développée au milieu du XIX'e siècle. Le Français Gaspard-Félix Tournachon, dit Nadar, prend en 1858 la première photo aérienne d'un quartier de Paris depuis un ballon dirigeable fixe. En 1860, James Wallace Black prend la première photo aérienne des États-Unis, depuis un ballon situé à 600 mètres d'altitude au-dessus de Boston (Info-Histoire, 2015).

Quant à la photographie satellitaire, elle naît avec la conquête spatiale dès 1957. La télédétection, discipline scientifique qui regroupe l'ensemble des connaissances et des techniques utilisées pour l'observation, I'analyse, l'interprétation et la gestion de l'environnement à partir de mesures et d'images obtenues à l'aide de plates-formes aéroportées, spatiales, terrestres ou maritimes (Université de Sherbrooke, s.d.), se greffe alors à la géographie (Institut numérique, 2014). Les satellites, comme LANDSAT (1972), sont équipés de caméras et d'appareils photographiques qui «mesurent» les ressources terrestres.

Ces technologies de prises de vues, aériennes ou spatiales, sont des sources primaires pour réaliser ensuite des cartes contenant des détails de plus en plus précis (grâce au pouvoir de synthèse et de répétition des prises de vue des satellites), que l'ordinateur permettra de représenter (Minelle, 1992, p. 104-107), à l'aide de systèmes d'information géographique (SIG). La production de ces cartes marque les débuts de la production de documents numériques (Minelle, 1992, p. 111-112).

\subsection{Les relevés, statistiques et autres études de terrain}

D'après le géographe Paul Claval, l'étude géographique étant une science de l'observation, la pratique du terrain revêt une importance particulière. En effet, le travail de terrain permet d'avoir une vue d'ensemble de ce que l'on étudie (2012). En outre, «le terrain ne se limite pas à I'analyse des paysages; il implique des visites, des entrevues » (Claval, 2007). 
Les documents produits par les études de terrain en géographie seront donc plus variés que des cartes: il peut s'agir de textes résultant d'entrevues, d'observations ou même de correspondances. Ces textes peuvent par ailleurs être établis par d'autres chercheurs (Claval, 2012). C'est pourquoi les textes anciens et les récits de voyage, tels que les écrits de Champlain ou de Darwin, par exemple, ont eu un impact certain dans l'étude géographique de la Nouvelle-France ou de l'Amérique du Sud.

Ces documents peuvent aussi être produits par des organismes gouvernementaux. II en va ainsi des statistiques élaborées à partir des recensements de population. Le géographe qui étudie l'évolution de la population du Québec pourra se servir de documents d'archives présentant, par exemple, les premiers recensements de la Province, que I'on peut voir à la Bibliothèque du parlement d'Ottawa.

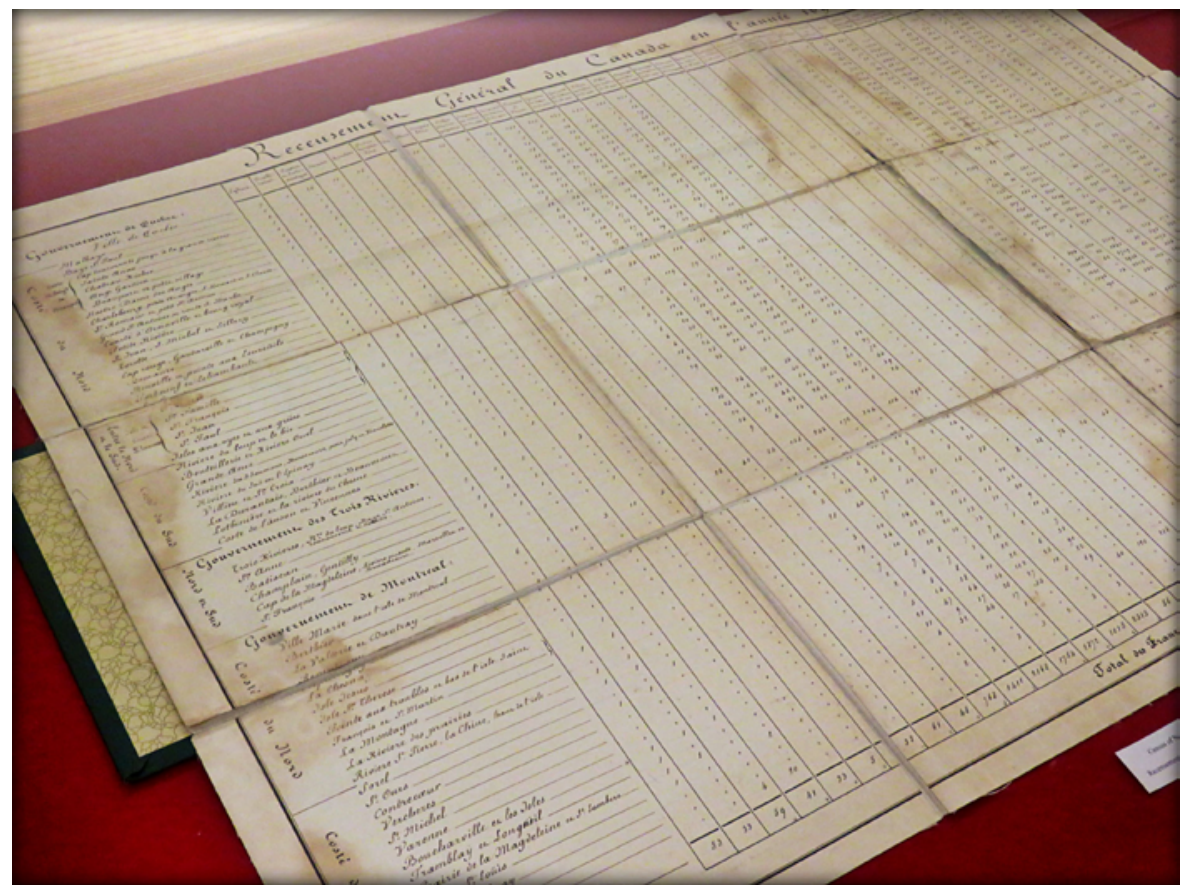

Figure 1: Le premier recensement effectué en Nouvelle-France - 1695. Source: Lætitia Le Clech. 


\section{LE TRAITEMENT DES ARCHIVES GÉOGRAPHIQUES}

En dépit du fait que les services d'archives traitent toutes les cartes de façon similaire même si l'information véhiculée dans les différents types de cartes diffère (Couture, 1994, p. 219), il existe quelques particularités aux documents cartographiques quant à leur traitement et leur conservation.

\subsection{Les conditions de conservation}

Les cartes géographiques peuvent faire partie intégrante d'un fonds d'archives, ou permettre la compréhension de documents qui les accompagnent. C'est pourquoi on les conservera en tant que telles (Rousseau et Couture, 1994, p. 219).

Dans un service d'archives, les cartes et les plans sont classés par région géographique, tandis que les plans architecturaux le sont par projet. Ces documents sont souvent conservés séparément des autres documents appartenant au fonds pour des raisons de dimensions. Pour maintenir un lien intellectuel entre la carte et le reste du fonds d'archives, il faudra alors utiliser une cote commune (système de renvois) (Couture, 1994, p. 220). Voici quelques exemples.

\subsubsection{L'exemple du Québec}

Lors d'une entrevue réalisée par l'auteure dans le cadre d'un travail universitaire, le cartothécaire de Bibliothèque et Archives nationales du Québec (BAnQ), Jean-François Palomino, explique que tout document cartographique publié au Québec est intégré d'office aux collections patrimoniales, par le mécanisme du dépôt légal, pour y être conservé de façon permanente. D'autre part, certaines cartes sont ainsi intégrées dans les fonds d'archives lorsqu'elles sont associées à une activité particulière en lien avec ce fonds. II n'y a pas de calendrier de conservation propre aux documents cartographiques. Ceux-ci sont intégrés en même temps que tous les autres documents associés (rapports, dossiers, photos, etc.). En effet, «les documents doivent être rattachés à l'unité de classification à laquelle ils correspondent selon leur contenu et le contexte de leur création, jamais en fonction de leur support » (Couture et al., 1999, p. 244).

Cependant, comme pour tout autre document imprimé, une carte peut être détachée du fonds si elle ne porte pas de marques particulières qui 
I'obligeraient à y rester. Elle peut être conservée séparément, en particulier pour des raisons de dimensions. II arrive donc que des documents cartographiques soient transférés dans la collection patrimoniale d'imprimés (Palomino, 2016).

\subsubsection{L'exemple de la Tunisie}

La majorité des documents cartographiques tunisiens sont conservés aux Archives nationales de Tunisie. Ils résultent de l'activité du gouvernement, en particulier durant la période coloniale (avant 1956). Cependant, les Archives nationales ne renferment pas de fonds purement cartographiques: ces documents sont conservés majoritairement dans le fonds de la période coloniale.

La Tunisie a mis en place un logiciel appelé SIGA (Système intégré de gestion des archives) permettant de décrire chaque document selon son type. Les documents cartographiques peuvent donc maintenant être décrits de façon unique, selon leur spécificité de création et leur situation de conservation, avec une référence archivistique propre et un système de renvois pour les documents qui constituent des pièces jointes à d'autres documents archivistiques (Hadhili, 2008).

\subsubsection{L'exemple de la France}

En France, le service des Cartes et Plans des Archives nationales a été créé en 1954. II est responsable d'une grande partie des documents cartographiques conservés au sein de l'institution, mais il faut noter que de nombreux documents se trouvent encore dans d'autres fonds des Archives nationales, notamment dans le fonds de la Marine.

Le service gère plusieurs séries: notamment la série $N$, classée par départements (16 000 pièces des XVII et $\mathrm{XVIII}{ }^{\mathrm{e}}$ siècles, concernant principalement Paris, la région parisienne, les pays du Nord de la Loire, ainsi que les pays annexés par l'administration napoléonienne), et le fonds des Travaux publics (15 000 pièces du XVII au XIXe siècle avec les atlas de Trudaine et Perronet [1754-1780]).

Outre les Archives nationales, d'autres institutions conservent un grand nombre de documents cartographiques, telles que la Bibliothèque historique de la Ville de Paris, la cartothèque de la bibliothèque interuniversitaire 
scientifique Jussieu, la Bibliothèque nationale de France, la cartothèque du centre de géographie, la cartothèque de l'Institut géographique national (IGN), les services historiques de l'armée de terre et de la marine, etc. (Comité français de cartographie, 1996).

\subsection{Les difficultés}

\subsubsection{Le respect des droits d'auteur}

II est parfois difficile de déterminer qui est l'auteur d'un document cartographique ou géographique, surtout si celui-ci n'a pas été produit par le gouvernement ou par une institution officielle. Parfois, les cartes conservées existent encore comme objet commercial et il est alors impossible de les rendre disponibles au grand public. Plusieurs bibliothèques et institutions ont cependant décidé d'utiliser des licences "Creative Commons», permettant un accès plus simple aux archives. Le gouvernement du Canada, de son côté, a établi une «Licence du gouvernement ouvert», qui permet «de copier, de modifier, de publier, de traduire, d'adapter, de distribuer ou d'utiliser autrement l'Information, quel que soit le support, mode ou format employé, à toutes fins légitimes» (Gouvernement du Canada, 2016), sans oublier, bien sûr, de mentionner la référence du document utilisé. Cette licence concerne par exemple les archives cartographiques que I'on trouve sur le site GéoGratis de Ressources naturelles Canada.

\subsubsection{La numérisation des cartes}

Les documents cartographiques, en général, sont difficiles à numériser en raison de leur taille et de la précision des informations qu'ils contiennent. Pour obtenir une bonne qualité d'image numérique, il a fallu établir des normes spécifiques pour ce type de document. Pierre-Yves Duchemin, qui fut conservateur à la Bibliothèque nationale de France, nous présente le processus de numérisation, prévu en 1994 à la BnF, de 300000 «images», à une résolution de 2048 par 3072 points par pouce. On savait cette résolution insuffisante pour une bonne utilisation d'images numérisées à partir de documents cartographiques (lecture satisfaisante des toponymes et des détails graphiques): ils ont finalement été numérisés à une résolution de 4000 par 6000 points par pouce (Duchemin, 2002). 
Plus récemment, Bibliothèque et Archives nationales du Québec, en collaboration avec la Bibliothèque nationale de France et le Musée canadien de l'histoire, a proposé un recueil de règles de numérisation (2015) dans lequel des procédures et des consignes très précises concernant les documents cartographiques sont définies à la page 28. Ainsi, la résolution demandée est de 600 ppp au minimum. D'autres consignes permettent de respecter l'intégrité de ces documents souvent très fragiles, telles que des spécificités concernant les couleurs (24 bits RVB Adobe RGB 1998) et une définition d'au minimum 4000 pixels pour le côté le plus long. Selon le format ( $A 0, A 1, A 2, A 3$ ou $A 4)$, la définition variera de manière proportionnelle, tandis que la résolution restera toujours de 600 points par pixel (Bibliothèque et Archives nationales du Québec, Bibliothèque nationale de France et Musée canadien de I'histoire, 2014, p.28).

Le projet Cartesius, fruit d'une collaboration entre les Archives de I'État en Belgique, I'Institut géographique national belge, la Bibliothèque royale de Belgique et le Musée royal d'Afrique Centrale, ouvre au public et aux scientifiques la plus grande collection de cartes et photographies aériennes de Belgique et d'Afrique Centrale. Ce portail sert également de base pour partager des expériences, des connaissances et des compétences spécifiques.

Les collections renferment des cartes anciennes, très belles et remarquables, souvent ignorées ou méconnues. Une part importante des collections cartographiques a été numérisée, mais il reste encore beaucoup de pain sur la planche, car ce travail est un processus de longue haleine (Cartesius, 2015).

\subsubsection{L'interprétation rendue difficile par une certaine fragilité}

Les cartes anciennes sont souvent des documents fragiles et uniques qu'il faut manipuler avec soin. Afin d'interpréter des archives géographiques et cartographiques, il est également nécessaire d'avoir des connaissances dans ces domaines. Comme nous avons pu le voir, les termes sont parfois très techniques et il existe un certain nombre de «codes», présents sur les cartes par exemple, qui en facilitent I'interprétation pour qui les connaît. En bref, pour se pencher sur les archives géographiques, il faut connaître la cartographie et l'intégrer à I'archivistique (Chebbi, 2016a). 


\section{L'UTILISATION DES ARCHIVES GÉOGRAPHIQUES}

\subsection{Pour la recherche historique}

\subsubsection{La reconstitution du passé géographique}

L'historien Charles Higounet a déclaré :

La géographie historique peut s'entendre aujourd'hui comme la restitution à un moment donné d'un état géographique qui a pu échapper d'ailleurs aux hommes de cette époque. C'est la reconstitution du passé géographique. (Higoune, 1961)

Pour le géographe Jean-René Trochet, c'est l' « étude des relations entre les hommes et les territoires dans les sociétés traditionnelles»(2000).

Les archives géographiques permettent de comparer et ainsi de comprendre l'évolution des espaces et des populations qui l'occupent. Elles permettent surtout d'étudier les paysages et les modes de vie à une époque donnée. Par exemple, dans les ressources cartographiques de BAnQ, on peut observer de nombreuses cartes de la Nouvelle-France qui nous renseignent entre autres sur la présence des populations autochtones.

Concernant la Ville de Montréal, la collection de Bibliothèque et Archives nationales du Québec (BAnQ) regorge de plans d'assuranceincendie, dressés par l'ingénieur Charles Goad dans le but d'aider les compagnies d'assurances à évaluer les risques d'incendie. Ces plans sont devenus très précieux: de nombreux chercheurs les consultent régulièrement dans les bibliothèques et dépôts d'archives partout au Canada et en particulier à Montréal. On y trouve une foule d'indications sur le mode d'aménagement de petites et grandes villes canadiennes de la fin du XIX et du début du XXe siècle (Buchanan et Gad, 2003). Consulter ces documents nous permet, par exemple, de préparer un voyage en situant des lieux que nous aimerions voir, de connaître l'histoire de l'endroit que l'on visite, de repérer des sites potentiellement contaminés (lors de l'achat d'un terrain ou d'une maison), de trouver des informations sur le lieu de passage ou de résidence d'un ancêtre ou d'un personnage historique, etc. 


\subsubsection{La géohistoire et la métagéographie}

La géohistoire, développée par le géographe Fernand Braudel, permet de comprendre les permanences et les dynamiques sociales en interaction avec leur géographie. La métagéographie, de son côté, s'intéresse à "l'ensemble des structures spatiales à travers lesquelles les individus et les sociétés ordonnent leur connaissance géographique du monde » (Pelletier, 2009).

Tel que le précise le géographe français Albert Demangeon, «pour expliquer les phénomènes géographiques dont l'homme a été le témoin ou l'artisan, il est nécessaire, à l'aide de documents d'archives, d'étudier leur évolution dans le passé » (1907, p. 193). En effet, un peu comme pour la géographie historique, les archives géographiques et cartographiques serviront à étudier le passé.

Pour lui, «l'intelligence de nombreux phénomènes géographiques est impossible si, à l'observation de leur état présent, on n'ajoute pas le tableau de leur évolution dans le passé »(Demangeon, 1907, p. 203).

Les archives géographiques utilisées dans un but historique peuvent faire l'objet d'expositions, comme par exemple celle de BAnQ Ils ont cartographié l'Amérique en 2008, ou encore constituer le matériau de base d'ouvrages historiques.

\subsubsection{La géopolitique}

«La géographie, ça sert, d'abord, à faire la guerre» (Lacoste, 1976).

Cette célèbre maxime, entendue par des milliers d'étudiants en géographie, redonne son importance à la géopolitique, qui avait été délaissée après la Seconde Guerre mondiale.

Cartographier le territoire est une nécessité et un gage de réussite militaire pour des États belligérants. De plus, l'État est bien souvent un des rares acteurs ayant les moyens humains et techniques suffisants pour établir et actualiser ce type de carte (Satgé, 2014).

Dans le domaine géopolitique, les archives cartographiques peuvent nous révéler les techniques utilisées durant certaines guerres. Par exemple, lors de la guerre du Viêt Nam, les cartes ont révélé que les Américains 
avaient bombardé des digues, provoquant inondations et assèchements de puits et de rivières, dans le but explicite de faire fuir les habitants afin qu'ils se regroupent et facilitent les offensives.

La conservatrice Marie-Anne Corvisier-de Villèle, qui s'est penchée sur la cartographie réalisée par l'armée de Terre française, part du constat que les forces militaires françaises possèdent un grand nombre de documents cartographiques, et ce, depuis la fin du XVII siècle, grâce à la mise en place par Louis XIV du Dépôt de la guerre. Ces documents cartographiques sont de trois types: des sources primaires, rédigées directement par les militaires (cartes et plans architecturaux de bâtiments); des sources cartographiques de toute provenance qui aident à la compréhension du terrain et à l'élaboration de stratégies; et enfin des documents cartographiques de conflits passés, utilisés pour apprendre des événements et contribuer à la formation des militaires (2001).

Le Dépôt de la guerre s'est transformé en 1880 en Service historique de l'armée de terre (SHAT), devenant un dépôt d'archives. Certains fonds cartographiques ainsi que certaines cartes ont été transférés à I'Institut géographique national (IGN) - autre entité civile créée en 1880 - en raison de leur grande utilité (cartes de Cassini, Cartes de l'État-major). Le SHAT conserve cependant «les travaux préparatoires, les levées et les mémoires qui les accompagnent, toute cette documentation cartographique ayant été considérée lors du partage comme "matière $d^{\prime}$ archives» n'ayant plus qu'une valeur historique » (Corvisier-de Villèle, 2001).

Ces archives cartographiques, établies par l'armée, sont aujourd'hui consultées par des historiens du paysage et de l'environnement. On constate donc encore une fois un usage historique des archives géographiques.

\subsubsection{Les cartes comme outils de propagande}

Selon le spécialiste Philippe Rekacewicz, «le cartographe invente une vision du monde» (2009). Les archives cartographiques peuvent nous renseigner sur les volontés politiques de certains États. II suffit pour cela d'avoir l'œil aiguisé et rompu aux symboles utilisés sur les cartes: point central autour duquel est ordonnée la projection de la carte, représentations cartographiques d'États vus comme indépendants 
alors qu'ils ne le sont point ou encore présence ou absence de certains éléments, tels que les routes. La carte, sans une certaine éthique, peut facilement devenir un objet de manipulation (Rekacewicz, 2009). On en trouve des exemples dans l'histoire de pays tels que la Palestine et Israël, les pays des Balkans, la Chine, etc. Étudier les archives cartographiques de ces pays permet de lire une histoire tourmentée. Sur les cartes, les noms de certains pays peuvent situer politiquement l'institution productrice de la carte: c'est le cas, par exemple, du Myanmar (nom imposé par la junte militaire), nommée ailleurs Birmanie, ou de la Mer de l'Est (pour la Corée du Sud), appelée Mer du Japon par les Japonais (Rekacewicz, 2006).

\subsection{Pour établir des politiques environnementales}

À la croisée de la géographie physique et de la géographie sociale, la géographie environnementale a pris de l'ampleur dans les dernières décennies avec des thématiques telles que le développement durable, la mondialisation et les concepts de changements climatiques et de réchauffement de la planète. Le géographe utilisera des archives (photos, cartes, rapports, données statistiques) afin de comparer les environnements étudiés. Cela lui permettra d'établir des normes, des exemples, et d'étudier les changements qui se produisent dans le monde.

\subsubsection{Les données climatiques et environnementales}

En climatologie, on utilise les archives depuis longtemps pour fournir des données aux scientifiques, qui peuvent ainsi établir des normes.

Le site météorologique du gouvernement du Canada offre ainsi des données et des cartes très intéressantes, permettant de comparer le climat d'aujourd'hui avec les années précédentes. On peut apprendre ainsi quelles sont les valeurs records et lire des statistiques élaborées par des climatologues et géographes. Le site de Météo-France, de son côté, offre la possibilité de faire une recherche sur le temps qu'il faisait en France à n'importe quelle date postérieure à 1966 (on voit le lien avec les données offertes et enregistrées par les satellites). Le champ des possibles est large, que ce soit pour le divertissement, l'étude ou autres.

D'un point de vue environnemental, les institutions appropriées peuvent se servir d'études géographiques pour bâtir des programmes et compiler 
des statistiques, afin d'anticiper des problématiques, voire des catastrophes (données sismologiques par exemple). On peut penser à la cartographie de la région arctique, par exemple, démontrant clairement l'impact du réchauffement climatique.

\subsubsection{Des données utiles pour faire des comparaisons}

Afin d'établir un état des lieux d'une situation environnementale, il peut être important de la comparer à ce qu'elle était auparavant. Pour cela, on peut utiliser des archives considérées comme géographiques (cartes, textes, photographies) pour montrer une évolution.

Par exemple, dans l'arrondissement LaSalle, de la Ville de Montréal, le Comité parc des Rapides cherche à montrer la dégradation de la qualité de l'eau au Québec. Pour cela, ses membres ont produit un rapport que l'on peut consulter et qui contient des archives gouvernementales, des articles de journaux, des relevés hydrographiques et des comptes rendus afin de présenter au Bureau d'audiences publiques sur l'environnement (BAPE) un bilan complet sur le sujet (Comité parc des Rapides Inc., 2000).

\section{LES ARCHIVES GÉOGRAPHIQUES À L'ÈRE NUMÉRIQUE}

\subsection{La numérisation et la pérennité}

Les quinze dernières années ont vu se développer le numérique: «la cartographie se transforme dans ses modes de consultation, de diffusion et de conservation »(Palomino, 2001). Les bibliothèques et archives nationales, dans de nombreux pays, ont amorcé de vastes programmes de numérisation des documents d'archives, en particulier des documents cartographiques, afin de les rendre disponibles au plus grand nombre (chercheurs, professeurs, étudiants, mais aussi grand public) et offrent maintenant sur leurs sites Internet leurs collections cartographiques presque complètes, permettant le regroupement de celles-ci et leur consultation depuis un seul endroit.

C'est le cas de la Bibliothèque nationale de France $(\mathrm{BnF})$ qui a été un précurseur en la matière puisque le programme de numérisation a débuté en 1994 (Duchemin, 2001, p. 68). Mais cette accessibilité pose 
plusieurs questions, notamment en ce qui a trait aux lois sur les archives, aux métadonnées (comment archiver sur Internet?) et aux données ouvertes (sécurité).

Les projets « d'archivages du Web» sont nombreux dans tous les pays. Mais « des choix s'imposent, et des pertes de contenus sont à accepter » (Chebbi, 2016b).

\subsubsection{L'obsolescence rapide des formats et des supports}

On sait que les formats numériques ne sont pas garants de longévité, il faut donc penser à la pérennité des archives. Les supports changent et les logiciels évoluent. De plus, pour ce qui est des documents cartographiques, il s'agit de fichiers très lourds. Ce n'est pas aussi simple d'archiver des cartes que des textes de revues savantes. II faut, pour pallier cette difficulté, prendre en charge le document dès sa création (Dubois et Vionnet, 2015). Afin de conserver les documents, de les rendre accessibles et de les garder intelligibles, il est donc important d'en faire un archivage pérenne. Ce sont, entre autres, les métadonnées qui en feront la description pour en permettre la compréhension sur le plus long terme possible. Mais le fait d'encadrer les processus d'archivage des documents cartographiques permettra également d'établir des normes visant la cohérence et la pérennité.

\subsubsection{Des normes à établir}

Devant la fragilité des contenus numériques, que les producteurs ne prennent pas toujours la peine de conserver, il est important d'établir des normes de conservation, notamment pour les contenus cartographiques. Jean-François Palomino explique:

... quelques institutions étrangères ont déjà fait face au problème. En Angleterre, par exemple, une entente a été conclue entre l'Ordnance Survey (agence gouvernementale productrice de cartes) et les différentes bibliothèques dépositaires, dont la British Library. Chaque année, un cliché des bases de données cartographiques est pris et déposé dans ces bibliothèques. Les bases de données peuvent être consultées par les usagers, mais il est strictement défendu d'en télécharger le contenu. (2001) 
De manière générale, il faut établir «une politique de conseil et de soutien en matière de gestion des documents papier ou électroniques auprès de leurs producteurs» (Dubois et Vionnet, 2015).

Devant des contenus toujours plus importants, «la gestion de documents spécialisés demande un personnel tout aussi spécialisé sachant bien s'adapter aux nouvelles réalités pour rassembler, ordonner et diffuser l'information » (Palomino, 2001). Malheureusement, on assiste aujourd'hui à une logique inverse avec des coupes importantes dans ces postes spécialisés.

\subsection{Les métadonnées et les données ouvertes}

Les métadonnées servent à archiver correctement les documents numériques. Géosource donne cette définition des métadonnées:

Liste structurée d'informations qui décrivent les données ou les services (incluant les données numériques ou non) stockés dans les systèmes d'information, les métadonnées peuvent contenir une brève description sur le contenu, les objectifs, la qualité et la localisation de la donnée ainsi que les informations relatives à sa création. (2012)

Obtenir de bons résultats de recherche dépendra de leur pertinence. En effet, chaque institution qui produit des documents cartographiques peut avoir son propre langage de description. Heureusement, il existe des standards, comme les normes ISO ou d'autres modèles plus spécifiques. Par exemple, le ministère des Ressources naturelles du Québec est un grand producteur de documents cartographiques; pour mieux les gérer et les conserver, il a mis en place le Programme d'identification cartographique (PIC) en 2004, y intégrant des informations sur les métadonnées numériques en 2007. Ainsi, les producteurs de cartes de ce ministère se basent sur le même document de référence pour dessiner leurs cartes. Le but de ce cadre de référence est, entre autres, de «faciliter l'utilisation des cartes pour les usagers en s'inspirant des bonnes pratiques en cartographie et en édition Web» (Ministère des Ressources naturelles, 2010).

La numérisation des documents cartographiques permet le libre accès à ces documents. Le développement de l'informatique et la diffusion d'Internet ont permis cette explosion des métadonnées cartographiques. 
Butch Lazorchak, un responsable de projets en technologies de I'information à la Bibliothèque du Congrès, trouve «difficile d'imaginer aujourd'hui un monde sans cartes» (2015, traduction libre). Cependant, il constate que la vaste majorité de l'information ne possède pas de métadonnées géographiques, ce qui est un non-sens alors que des applications comme Google Maps existent.

En 2013, le projet Global Database of Events, Language, and Tone (GDELT) est lancé, élargissant les possibilités offertes par les métadonnées pour cartographier le monde. En effet, le projet permet de représenter des cartes des conflits dans le monde en faisant des mises à jour quotidiennes et en se basant sur des archives de l'actualité internationale des 30 dernières années. Le projet est impressionnant, mais montre les limites du big data qui manque parfois de précision (Lazorchak, 2015).

\subsection{L'accessibilité aux archives géographiques et la créativité citoyenne}

\subsubsection{Le site Google Maps}

La création de Google Maps en 2004 aux États-Unis a largement contribué à la démocratisation de l'utilisation des cartes par les citoyens.

Le principe de ce service de cartographie en ligne, aujourd'hui connu de presque tous, est basé sur l'exploitation de données et d'images aériennes et satellites pour créer des cartes sur lesquelles I'utilisateur peut zoomer avec une grande précision et variété.

D'autres services de cartographie se sont créés au même moment ailleurs, tels qu'OpenStreetMap, créé par l'anglais Steve Coast en 2004, qui offre un peu les mêmes fonctionnalités que Google Maps, mais en y ajoutant une touche créative, puisque le logiciel est une base de données cartographiques ouvertes, fonctionnant avec des données appelées Global Positioning System (GPS). Ce type de service cartographique, par la facilité des mises à jour pouvant être faites, peut avoir des applications très concrètes. Par exemple, lors du tremblement de terre en Haïti en janvier 2010, des cartographes en herbe ont effectué plus de 800 changements sur la carte de Port-au-Prince; le plan de la ville est devenu très précis, facilitant le repérage et l'efficacité des secours. Cette mise à jour a pu 
se faire en consultant des archives cartographiques, en l'occurrence celles de la CIA, ainsi que des données fournies par d'autres services de cartographie en ligne, telles que celles de Yahoo!

Le projet s'est organisé très rapidement grâce aux réseaux sociaux et aux moyens collaboratifs dont nous disposons sur Internet aujourd'hui (Gévaudan, 2010).

\subsubsection{Les applications mobiles et Internet}

Plusieurs applications mobiles portant sur la cartographie ont été développées ces dernières années. Celles-ci proposent à l'utilisateur de découvrir son quartier, ou bien encore des éléments historiques de la ville qu'il est en train de visiter durant ses vacances, dans une perspective historique, en consultant des cartes anciennes. II en va ainsi du projet Old Maps Online, qui combine un puissant moteur de recherche à la technologie de Google Maps. Près de 400000 cartes anciennes provenant de 33 institutions du monde entier ont été indexées et centralisées pour les rendre accessibles à tous depuis un seul site Internet (Jost, 2015a). L'Université américaine de Yale a réalisé de son côté un travail archivistique combinant photographie et cartographie: explorer les États-Unis tels qu'ils étaient entre 1935 et 1944, durant la Grande Dépression, à l'aide de plus de 170000 photos d'archives organisées sur une carte interactive. Ce système fonctionne grâce à la géolocalisation des photographies qui ont été prises dans le cadre d'un programme gouvernemental lancé après la crise économique de 1929 (Jost, 2015b).

\subsubsection{La créativité citoyenne}

Avec l'accessibilité offerte par Internet, il est possible de s'approprier des archives cartographiques pour en faire des projets artistiques uniques. Ces temps-ci, on peut observer un certain engouement pour les cartes anciennes, pour leur beauté et pour l'information qu'elles nous offrent sur des époques révolues.

Quadrature, un collectif d'artistes basé à Berlin, utilise des données géospatiales pour tracer, à l'aide d'une machine qui recueille en temps réel la position de certains satellites, des trajectoires sur une vieille carte. Le projet Kartograph leur permet de recréer des cartes en superposant de nouvelles données à une carte ancienne. 


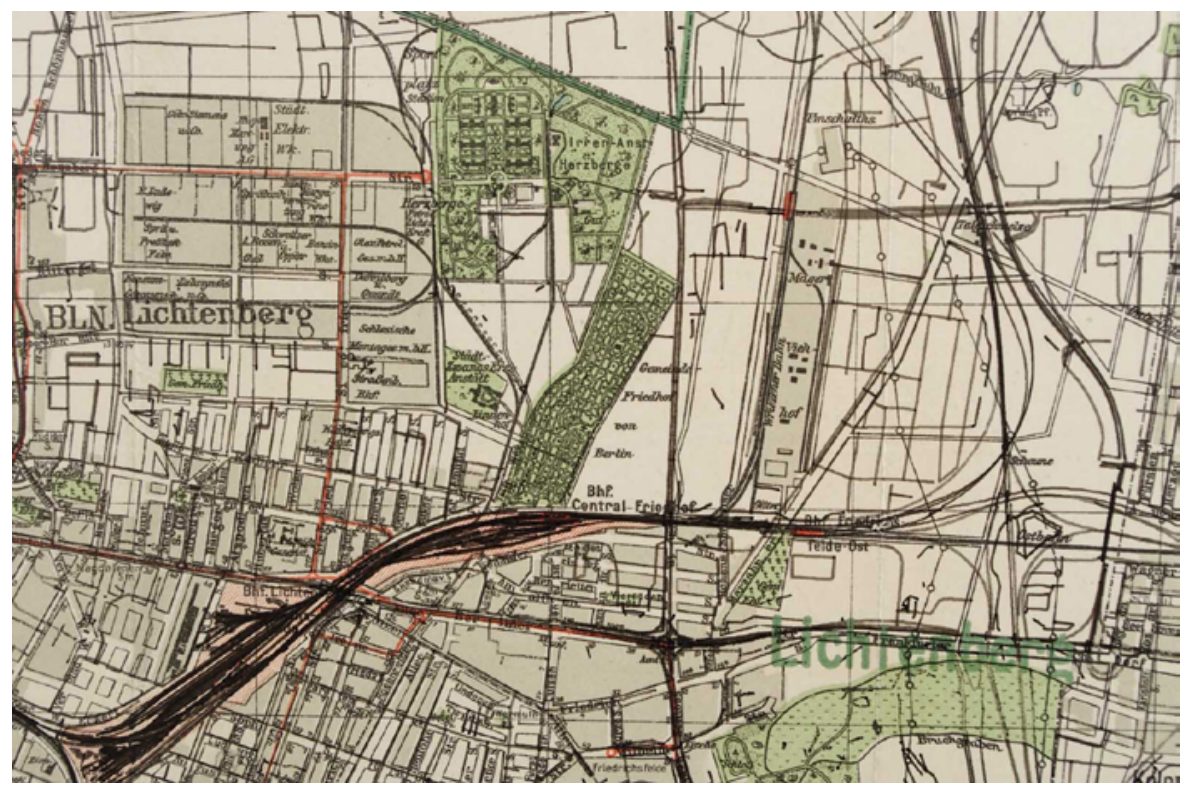

Figure 2: Projet Kartograph, 1938/2015 Berlin. Source: Quadrature (collectif).

Anton Dubrau, spécialiste montréalais du transport en commun, des cartes et des applications numériques, propose sur son site des modélisations cartographiques pour des projets reliés la plupart du temps au transport en commun. II a notamment réussi à superposer des cartes du transport en commun de Montréal en 1941 à celles d'aujourd'hui.

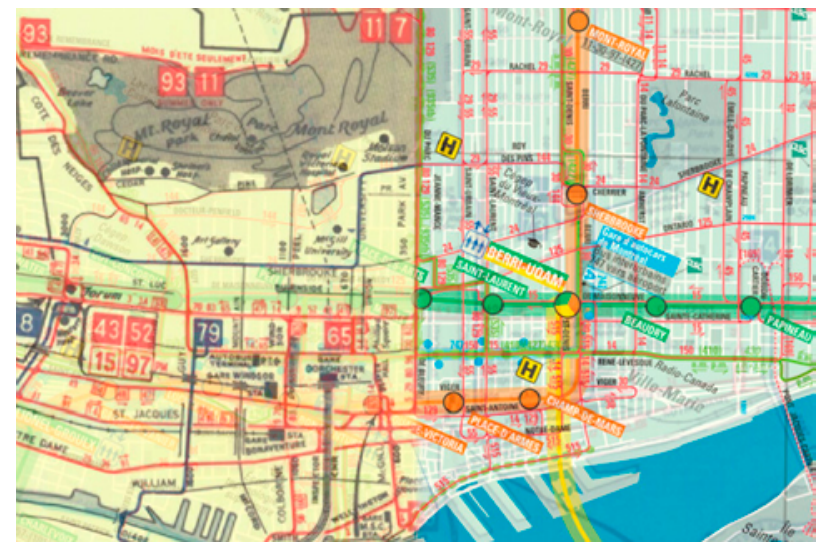

Figure 3: Montreal Transit Map 1941 vs 2011.

Source : Anton Dubrau, blogue Catbus 
Eric Fisher, un autre passionné de cartes, a cartographié les lieux les plus photographiés dans les grandes villes, selon que les photos étaient prises par des touristes (en rouge) ou des habitants de la ville (en bleu). Le résultat, magnifique, se rapproche de l'œuvre d'art.

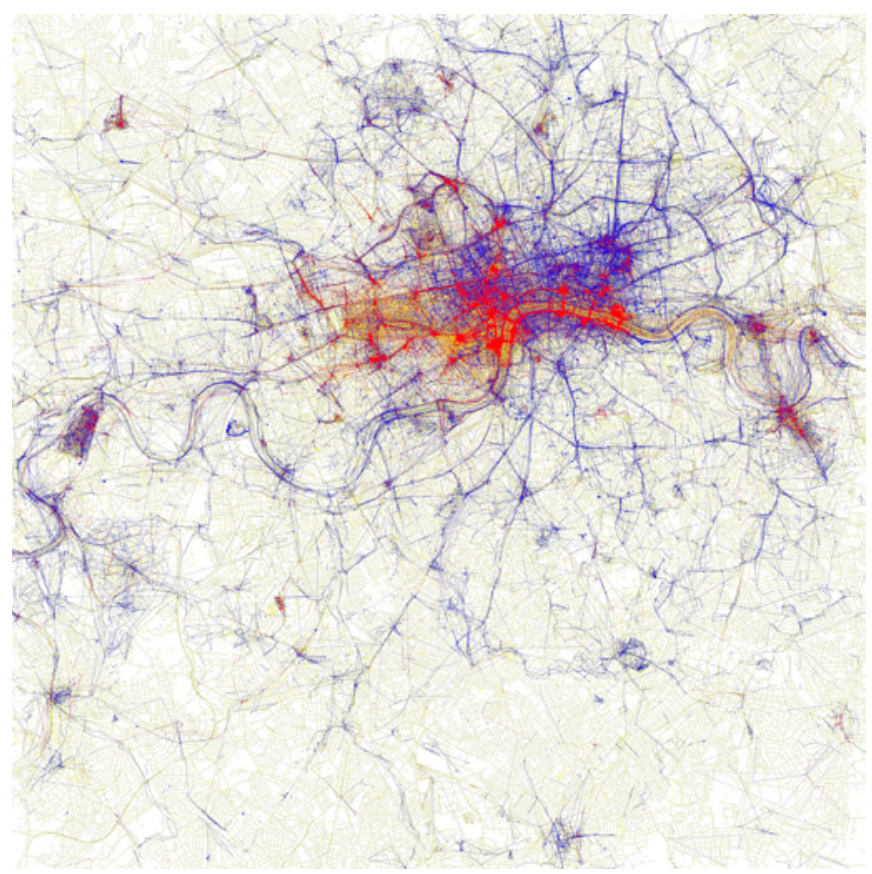

Figure 4: Local and Tourists : London

Source : Eric Fisher (Flickr/OpenStreetMap)

\section{CONCLUSION}

Se plonger dans la thématique des archives cartographiques peut nous entraîner dans un univers passionnant et varié. Le nombre de documents, de projets et d'idées qui se développent autour des cartes montre la vitalité de ce domaine que l'on pourrait penser menacé par I'arrivée du numérique. Comme le précisait déjà Jean-François Palomino (BAnQ) en 2001:

[l]es projets misent non seulement sur l'aspect esthétique, mais aussi et surtout sur l'importance des cartes anciennes 
pour l'enseignement et la recherche dans plusieurs disciplines, notamment la généalogie, la géographie historique, I'histoire de la cartographie, I'histoire environnementale, I'histoire urbaine, I'histoire culturelle, I'histoire des idées, etc. (Palomino, 2001)

Les archives géographiques doivent être prises en charge d'une manière plus encadrée pour éviter les détournements et pour assurer leur présence sur Internet. De cette manière, elles feront l'objet de travaux réellement originaux pouvant servir à modéliser des problématiques environnementales ou à étudier le fonctionnement de nos sociétés.

LAetitia Le CLeCH

\section{BIBLIOGRAPHIE}

\section{Notes de cours}

CHEBBI, A. (2016a). SCI6117 - Notes du module 2, Université de Montréal. [Cours en ligne]. Repéré dans l'environnement StudiUM: https://studium.umontreal.ca/

CHEBBI, A. (2016b). SCI6117 - Notes du module 10, Université de Montréal. [Cours en ligne]. Repéré dans l'environnement StudiUM: https://studium.umontreal.ca/

\section{Ouvrages}

CIATTONI, A. et VEYSRET, Y. (2010). Les fondamentaux de la géographie ( 2 édition). Paris: Armand Colin.

CLAVAL P. (2007). Épistémologie de la géographie ( $2^{\mathrm{e}}$ édition). Paris: Armand Colin.

COUTURE, C. et ROUSSEAU, J.Y. (1994). Les fondements de la discipline archivistique. Québec: Les Presses de I'Université du Québec. 
COUTURE et al. (1999). Les fonctions de l'archivistique contemporaine. Québec: Les Presses de I'Université du Québec.

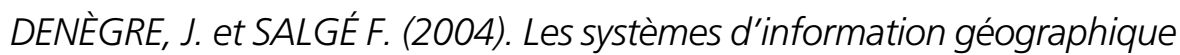
( $2^{\mathrm{e}}$ édition). Paris: Presses universitaires de France «Que sais-je? »

CHARLES HIGOUNET, C. (1961). La géohistoire. Dans Ch. Samaran (dir.), L'histoire et ses méthodes (68-91). Paris: Pléiade.

LE FUR, A. (2010). Pratiques de la cartographie ( $2^{\mathrm{e}}$ édition). Paris: Armand Colin.

MINELLE, F. (1992). Représenter le monde. Paris: Presses Pocket.

TROCHET, J.R. (2000). Géographie historique. Hommes et territoires dans les sociétés traditionnelles. Paris: Nathan.

\section{Entretien}

PALOMINO, J.-F. (2016, février). Les archives cartographiques à BAnQ propos recueillis par Laetitia Le Clech dans le cadre du cours SCI6117, Université de Montréal (entrevue avec un archiviste).

\section{Articles et études}

BIBLIOTHĖQUE ET ARCHIVES NATIONALES DU QUÉBEC. (s.d.). Collection patrimoniale de cartes géographiques. Repéré à http://www.banq. qc.ca/collections/collections_patrimoniales/collections speciales/ documents cartographiques/

BIBLIOTHÈQUE ET ARCHIVES NATIONALES DU QUÉBEC, BIBLIOTHÈQUE NATIONALE DE FRANCE et MUSÉE CANADIEN DE L'HISTOIRE. (2014). Recueil de règles de numérisation. Repéré à http://collections.banq. qc.ca/bitstream/52327/2426216/1/4671601.pdf

BUCHANAN, E. et GAD, G. (2003). Goad, Charles Edward. Dans Dictionnaire biographique du Canada, vol. 13. Université Laval/ University of Toronto. Repéré à http://www.biographi.ca/fr/bio/goad charles edward 13F.html 
CLAVAL, P. (2012, 1 avril). Le rôle du terrain en géographie. Confins 17. doi : $10.4000 /$ confins. 8373

COMITÉ FRANÇAIS DE CARTOGRAPHIE. (1996). Collections parisiennes de documents cartographiques. Repéré à http://www.lecfc.fr/new/ articles/149-article-12.pdf

COMITÉ PARC DES RAPIDES INC. (2000). Sur la gestion de l'eau du Québec. Extrait d'archives environnementales. Présenté à la Commission Beauchamp BAPE à Montréal le 23 novembre 1999. Repéré à http://www.strategiessl.qc.ca/pdf/memoire_gestioneau cprapides 199911 2.pdf

CORVISIER-DE VILLÈLE, M.-A. (2001). Les fonds cartographiques conservés au service historique de l'armée de terre: bref survol. Comité français de cartographie 167. Repéré à http://www.lecfc.fr/new/articles/167article-2.pdf

DEMANGEON, A. (1907). Les recherches géographiques dans les archives. Annales de Géographie, 16(87), 193-203. Repéré à http://www.jstor. org/stable/23437064

DUBOIS, A. et VIONNET, F. (2015, 8 décembre). Les Archives de l'État du Valais (Suisse) ou le défi de l'archiviste contemporain. Revue électronique suisse de science de l'information (RESSI). Repéré à http:// www.ressi.ch/num16/article 112

DUCHEMIN, P.Y. (2002). La numérisation des documents cartographiques anciens: supports traditionnels et nouvelles technologies. International Journal of Special Libraries (INSPEL) 36(1), 67-82. Repéré à http://forge. fh-potsdam.de/ IFLA/INSPEL/02-1dupi.pdf

GÉVAUDAN, C. (2010, 15 janvier). Haïti : Mobilisation autour d'une carte libre. Libération. Repéré à http://www. liberation.fr/ecrans/2010/01/15/ $\underline{\text { haiti-mobilisation-autour-d-une-carte-libre 950513?page=article }}$

GOUVERNEMENT DU CANADA. (2016). Gestion des documents cartographiques, architecturaux et techniques dans l'administration fédérale. Repéré à http://web.archive.org/web/20160817061416/http://www.baclac.gc.ca/fra/services/gestion-ressources-documentaires-gouvernement/ gestion-information/Pages/gestion-documents-cartographiques.aspx 
HADHILI, R. (2008). Traitement des cartes et plans.

INFO-HISTOIRE.COM. (2015). Les premières photographies de I'histoire. Repéré à http://www.info-histoire.com/15658/premieres-photos-histoire/

INSTITUT NUMÉRIQUE. (2014, 1 février). Histoire de la télédétection. Repéré à http://www.institut-numerique.org/22-historique-de-lateledetection-52eca9e7582a9

JOST, C. (2014, 9 avril). La New York Public Library met en ligne 20000 cartes d'archives téléchargeables gratuitement. Archimag. Repéré à http://www.archimag.com/archives-patrimoine/2014/04/02/ new-york-public-library-20000-cartes-archives

JOST, C. (2015a). Old Maps Online: 280000 cartes historiques gratuites sur votre smartphone. Archimag. Repéré à http://www.archimag. com/archives-patrimoine/2015/08/31/old-maps-online-consultezgratuitement-400-000-cartes-historiques

JOST, C. (2015b). Voyagez dans les photos d'archives de la Grande Dépression américaine depuis une carte interactive. Archimag. Repéré à http://www.archimag.com/archives-patrimoine/2015/10/09/photosarchives-grande-depression-americaine-carte-interactive

KIDD, B. (2003). Digitization of cartographic materials: National Archives of Canada. International Journal of Special Libraries (INSPEL) 36(1), 53-66. Repéré à http://forge.fh-potsdam.de/ IFLA/INSPEL/02-1kibe.pdf

LAZORCHAK, B. (2015). Mapping Words: Lessons Learned From a Decade of Exploring the Geography of Text [Billet de blogue]. Repéré à http://blogs.loc.gov/digitalpreservation/2015/04/mapping-wordslessons-learned-from-a-decade-of-exploring-the-geography-of-text/

MARTIN, G.J. (1964). Geographers and archives: a suggestion. The Professional Geographer 16(6), 25-27. doi: 10.1111/j.00330124.1964.025_z.x

MINISTÈRE DES RESSOURCES NATURELLES DU QUÉBEC. (2010). Programme d'identification cartographique (PIC) - Cadre de référence sur l'habillage. Repéré à http://www.quebecgeographique.gouv.qc.cal approfondir/bibliotheque/normes.asp 
PALOMINO, J.F. (2001). Cartographie et révolution numérique: la bibliothèque à l'ère électronique. Documentation et bibliothèques 47(3), 119-122.

REKACEWICZ, P. (2006). La cartographie, entre science, art et manipulation. Le Monde diplomatique 623, 14.

REKACEWICZ, P. (2009). Quand le cartographe invente la réalité... Manière de voir 107, 68.

SATGÉ, V. (2014, 28 juin). Y. Lacoste, «La géographie, ça sert d'abord à faire la guerre ». Éd. La découverte. Repéré à http://www.diploweb. $\mathrm{com} / Y$-Lacoste-La-geographie-ca-sert-d.html

STAGE TECHNIQUE INTERNATIONAL DES ARCHIVES. (2010). Supports audiovisuels et numériques. Repéré à http://www.archivesdefrance. culture.gouv.fr/static/3837

UNIVERSITÉ DE SHERBROOKE. (s.d.). Qu'est-ce que la télédétection? Repéré à https://www.usherbrooke.ca/geomatique/programmesdetudes/par-discipline/teledetection/quest-ce-que-la-teledetection/

WEBER-PALLEZ, C. (2013). Faire de la géographie historique en Grèce antique aujourd'hui [Billet de blogue]. Repéré à https://lagoradugotha. hypotheses.org/112

\section{Enregistrement vidéo}

REKACEVICZ, P. (2015). La cartographie: entre science, art et manipulation. Communication présentée dans le cadre de la conférence «La carte invente le monde ». Lille, France. Repéré à http://lille1tv.univ-lille1.fr/ collections/video.aspx?id=d6ff9593-ed24-4b20-9d2b-94bea5ec0ea9

\section{Sites Internet}

Le blogue d'Anton Dubrau: http://www.cat-bus.com/ Archives de Montréal : http://archivesdemontreal.com Association cartographique internationale (ACI / ICA) : http://icaci.org/ 
Association des cartothèques et archives cartographiques du Canada (ACACC) : http://www.acmla-acacc.ca/

Cartesius: https://www.cartesius.be/CartesiusPortal/

Collectif Quadrature (Projet Kartograph): http://quadrature.co/work/ kartograph/

Collection patrimoniale BAnQ de cartes géographiques: http://www. banq.qc.ca/collections/collections patrimoniales/collections speciales/ documents_cartographiques/

La galerie d'Éric Fisher (Locals and Tourists \#1 (GTWA \#2): London.) https://www.flickr.com/photos/walkingsf/4671589629/in/album72157624209158632/

GéoGratis (Ressources naturelles Canada): https://www.rncan.gc.ca/ sciences-terre/geographie/information-topographique/donneesgratuites-geogratis/11043

Géosource: http://www.geosource.fr/docs/introduction/introduction.html

Histoire de la photographie (Info-Histoire): http://www.info-histoire. com/15658/premieres-photos-histoire/

Ils ont cartographié l'Amérique (BAnQ) : http://www.banq.qc.ca/activites/ expositions/2008/expo decouverte amerique/index.html

Montréal historique: http://imtl.org/montreal_historique.php 